\title{
Computerized breast cancer analysis system using three stage semi- supervised learning method
}

\author{
Wenqing Sun ${ }^{\mathrm{a}}$, Tzu-Liang (Bill) Tseng ${ }^{\mathrm{b}}$, Jianying Zhang ${ }^{\mathrm{c}, \mathrm{d}}$, Wei Qian ${ }^{\mathrm{a}, \mathrm{d}}$
}

a. Department of Electrical and Computer Engineering, University of Texas at El Paso, 500 West University Avenue, El Paso, Texas, United States, zip code 79968

b. Department of Industrial, Manufacturing \& Systems Engineering, University of Texas at El Paso, 500 West University Avenue, El Paso, Texas, United States, zip code 79968

c. Department of Biological Sciences, University of Texas at El Paso, 500 West University Avenue, El Paso, Texas, United States, zip code 79968

d. Sino-Dutch Biomedical and Information Engineering School, Northeastern University, No.11, Lane 3, Wenhua Road, Heping District, Shenyang, Liaoning, China, zip code 110819

Corresponding author:

Wei Qian, Ph.D.

Professor

Department of Electrical and Computer Engineering

Director: Medical Imaging Informatics Laboratory

College of Engineering, University of Texas, El Paso

500 West University Avenue

El Paso, Texas 79968

$\mathrm{PH}:(915) 747-8090$

FX: (915) 747-7871

http://ee.utep.edu/facultyqian.htm

E-mail addresses: wqian@utep.edu 


\title{
Computerized breast cancer analysis system using three stage semi- supervised learning method
}

\author{
Wenqing Sun, Tzu-Liang (Bill) Tseng, Jianying Zhang, Wei Qian
}

\begin{abstract}
Background and Objective

A large number of labeled medical image data is usually a requirement to train a well-performed computer-aided detection (CAD) system. But the process of data labeling is time consuming, and potential ethical and logistical problems may also present complications. As a result, incorporating unlabeled data into CAD system can be a feasible way to combat these obstacles.
\end{abstract}

Methods

In this study we developed a three stage semi-supervised learning (SSL) scheme that combines a small amount of labeled data and larger amount of unlabeled data. The scheme was modified on our existing CAD system using the following three stages: data weighing, feature selection, and newly proposed dividing co-training data labeling algorithm. Global density asymmetry features were incorporated to the feature pool to reduce the false positive rate. Area under the curve (AUC) and accuracy was computed using 10 fold cross validation method to evaluate the performance of our CAD system. The image dataset includes mammograms from 400 women who underwent routine screening examinations, and each pair contains either two cranio-caudual (CC) or two mediolateral-oblique (MLO) view mammograms from the right and the left breast. From these mammograms 512 regions were extracted and used in this study, and among them 90 regions were treated as labeled while the rest were treated as unlabeled.

Results

Using our proposed scheme, the highest AUC observed in our research was 0.841, which included the 90 labeled data and all the unlabeled data. It was $7.4 \%$ higher than using labeled data only. With the increasing amount of labeled data, AUC difference between using mixed data and using labeled data only reached its peak when the amount of labeled data was around 60 .

\section{Conclusions}

This study demonstrated that our proposed three stage semi-supervised learning can improve the CAD performance by incorporating unlabeled data. Using unlabeled data is promising in computerized cancer research and may have a significant impact for future CAD system applications.

Key Words: Semi-supervised learning, computer aided detection, mass detection, unlabeled data 


\section{Introduction}

Breast cancer refers to the erratic growth of cells that originate in breast tissue and is one of the most common types of cancer [1]. Scientific evidences have shown that early cancer detection is important to enhance the survival rate of the patients through more effective patient management and treatment [2-3]. Mammography has been the most cost-effective and widely used imaging modality for breast cancer screening during last few several decades [4]. To help the radiologists diagnose early breast cancer, many methodologies have been implemented to improve the performance of computer aided detection (CAD) systems.

However, most of the existing CAD systems are implemented via supervised learning which is based on a large volume of labeled breast cancer image data. These data come with truth files (disease statuses marked by the radiologists such as "malignant" or "benign"), and to obtain them requires radiologists to read and mark the mammograms. The malignant masses are marked and labeled as malignant areas while the rest of the areas are considered as benign or normal. In CAD systems, features are usually extracted from region of interest (ROI), so every ROI is condensed into a vector of numerical numbers and the labels of corresponding areas will be paired with these features. Unfortunately, acquiring a large amount of labeled data is usually time consuming [5], and making diagnosis on such large number of cases is a heavy burden for radiologists. In order to label all the samples, several radiologists are required to evaluate all the data individually, compare their evaluations with one another, and conduct a discussion of the resulting analysis to reach a final conclusion. Moreover, confidentiality agreements are required to obtain the data, and typically neither the doctors nor the patients are willing to reveal this information. On the other hand, abundance of unlabeled data (no truth file related or "truth partially known") is likely to be more accessible in most research contexts and will significantly reduce the problem of confidential sensitivity [6].

One feasible way to solve this problem is to combine a small amount of labeled data with a large amount of unlabeled data to build the classifier together. In the area of machine learning, this technique is called semi-supervised learning (SSL). It exploits labeled data and unlabeled data at the same time without any human intervention. The SSL algorithm allows us to label some of the unlabeled data based on the information provided by the initial labeled dataset, and the newly labeled data (also called pseudo-labeled data [7]) can be used to improve the CAD performance. One popular and promising SSL method is called co-training, which is proposed by [8]. It trains two classifiers by letting each classifier label the unlabeled data for the other one, and it makes decisions based on the agreement of these two classifiers. Although the co-training method has been successfully used in many fields [9-11], the requirement of two sufficient and redundant feature subsets can be hardly met in CAD systems. In the breast cancer CAD area, some research groups have tried different SSL methods to utilize the unlabeled data. [12] extended the cotraining algorithm by incorporating the random forest ensemble method to determine the most confident examples to label. [6] made a comparison of the transductive dimension reduction method and semi-supervised LapSVM manifold regularization method with an ultrasound image database containing 1126 lesions. In our group, we have conducted a co-training based SSL breast cancer research based on labeled and unlabeled ROIs [13].

The need to avoid false positives cannot be over stressed for CAD systems since it might bring psychological harms, unnecessary imaging tests and biopsies in women without cancer and inconvenience [14-15]. Moreover, different from traditional supervised machine learning 
techniques, SSL methods have to overcome several other obstacles including the conflict of accuracy and diversity [16] as well as noisy data in labeled dataset [17]. Many efforts have been made to solve these problems. For example, to reduce the false positive rate, a number of computerized schemes have been reported and tested to segment fibroglandular tissue and compute mammographic density [18-20], and [4] found that the incorporation of global mammographic density measurements into a CAD system can help accomplish this goal. To create the diversity, [16] developed a method by manipulating the training set, and based on this theory they proposed the inter-training algorithm. To eliminate the noisy data, instant selection algorithms have been discussed and compared [17-18]. However, to the best of our knowledge, no mammogram based SSL scheme has been reported, and the possibility of redesigning and modifying the traditional supervised learning based CAD systems to semi-supervised learning CAD systems have never been investigated and tested.

The aim of this study is to investigate and test that whether redesigning CAD system and incorporating the modified SSL algorithm can efficiently use unlabeled data and thus improve the performance of detection result. For this purpose, a novel three stage semi-supervised learning approach was designed and tested based on our existing CAD system [19-21]. This system has been widely tested in the clinical practice to assist radiologists in reading and interpreting mammograms to date [22-23]. Our SSL method integrated data weighing, feature selection and a newly proposed dividing co-training data labeling method. The global density asymmetry features were integrated into the CAD system, and each module of the system was modified and redesigned to adapt to the proposed method. Dr. Wei Qian organized this study, and the data we used for this study was collected by Dr. Wei Qian, Dr. Tzu-Liang (Bill) Tseng and Dr. Jianying Zhang. The method was developed by Wenqing Sun and Dr. Wei Qian together.

\section{Materials and methods}

\subsection{Data}

From an established in-house full-field digital mammography (FFDM) image database, 400 cases were investigated and tested which included 200 pairs of cranio-caudual (CC) view and 200 pairs of mediolateral-oblique (MLO) view breast images, and each pair contains two mammograms acquired from both right and left breasts. The women's ages range from 32 to 68, with a mean age of 47.7 years and median age of 43 . Figure 1 shows the cases in positive and negative subgroups have similar distributions of subjectively rated mammographic density (i.e. Breast Imaging Reporting and Data System (BIRADS)). The majority of the cases (37.0\% and $52.0 \%$ ) were rated by radiologists as scattered fibro-glandular (BIRADS II), heterogeneously dense (BIRADS III). In this dataset, 200 are positive cases with cancer detected and verified by biopsy and pathology examinations and the other 200 were negative cases from routine screening mammography examinations. There are 3142 ROIs extracted from these 400 cases, and 221 out of the 3142 ROIs are diagnosed as malignant masses while the rest are diagnosed as normal tissues. Each ROI is composed of 256 by 256 pixels at 12-bit gray values. These 221 malignant masses were extracted from the 200 positive cases including one mass detected in each of 181 cases, 2 detected in each of 17 cases, and 3 detected in 2 cases. Among these 221 malignant masses there were 81 stellate, 48 circumscribed, and 92 irregular masses. To maintain the balance between the two classes of the data, only 512 ROIs were randomly selected in our research which included all the 221 abnormal ROIs and 291 normal ROIs. Table 1 shows 265 out of the 512 regions were from density ROIs, 101 from fatty ROIs, and 146 from mixed ROIs. 
Among the 291 normal ROIs, 91 were from positive cases (either normal tissue in the positive cases apart from the malignant masses or the corresponding contralateral normal breast), and 200 were from each of the negative cases. In this study all the 400 cases have used at least one ROI. All the data we used in this research are labeled data, and when the proposed algorithm was evaluated, the truth files (practitioner diagnosis) from some of the data were removed and treated as unlabeled data.

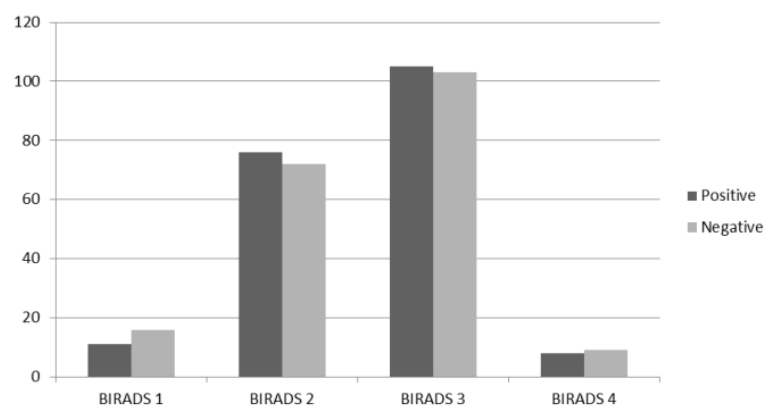

Figure 1: Distribution of breast density BIRADS ratings in the Database

\begin{tabular}{cccc}
\hline Mass Type & Stellate & Circumscribed & Irregular \\
\hline Mass Number & 81 & 48 & 92 \\
Percentage & 36.7 & 21.7 & 41.6 \\
\hline \hline & & & \\
\hline ROI Type & Density & Fatty & Mixed \\
\hline ROI Number & 265 & 101 & 146 \\
Percentage & 51.8 & 19.7 & 28.5 \\
\hline
\end{tabular}

Table 1: Distribution of 221 malignant masses and 512 ROIs in the database

\subsection{Proposed method}

In this study we proposed a three stage SSL method and we also investigated and tested the possibility of converting and integrating the traditional supervised learning based CAD system to the semi-supervised learning based CAD system. Figure 2 shows the flowchart of the proposed scheme. The techniques of preprocessing, region segmentation, and feature extraction can be found in [22-23]. All the labeled data and unlabeled data were applied to the preprocessing module [22] and all the parameters were set to be the same for every case. To get better performance of our proposed SSL algorithm, more features were developed and extracted from the ROIs in this study compared to our traditional CAD system [23]. Furthermore, the bilateral density asymmetry features calculated from the corresponding entire breast areas (global features) were combined with the features calculated from certain region of interest (local features). To increase the discriminative power of our semi-supervised CAD system, we combined the local and global features together. This is based on the fact that breast tissue density can be considered as a useful risk indicator of women having or developing breast abnormalities and cancers [2425]. And as reported by [4], incorporating automatically computed mammographic density features into CAD schemes enabled it to achieve better performance compared to the incorporation of manually (subjectively) assessed density. For all the extracted suspicious areas, the local features were calculated from the region itself and the global features were acquired 
from ratios of the whole right and left breasts depicted on the mammograms. A brief description of each feature is listed in Table 2. To the best of our knowledge, the global features 20-28 have never been used in breast cancer diagnosis.

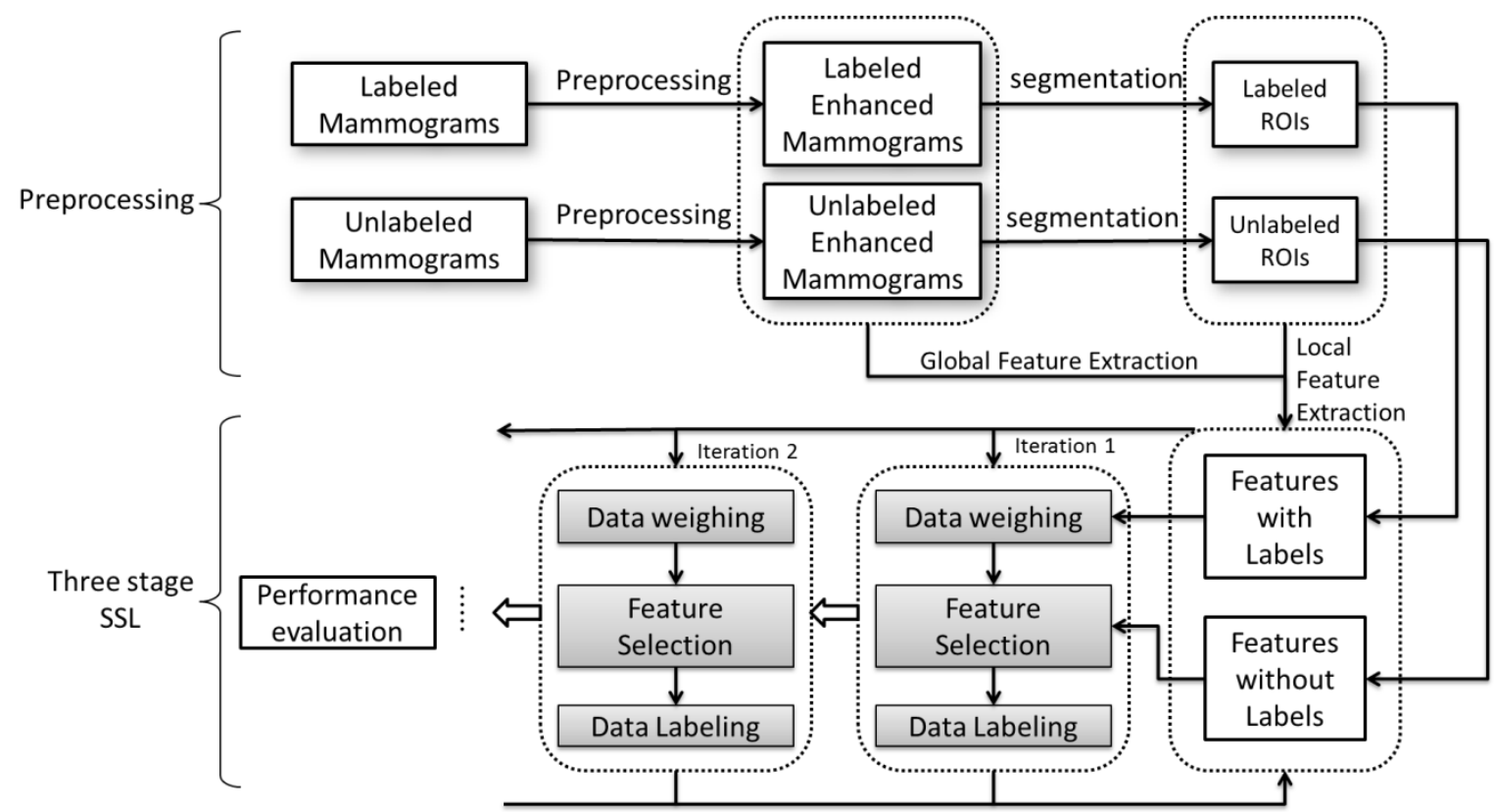

Figure 2: The flowchart of our proposed scheme. The first major section is called preprocessing stage and the second major section is called SSL stage. 


\begin{tabular}{|c|c|c|}
\hline Category & Number & Discriptions \\
\hline \multirow{19}{*}{$\begin{array}{c}\text { Local } \\
\text { Features }\end{array}$} & 1 & Circularity \\
\hline & 2 & Normalized deviation of radial length \\
\hline & 3 & Ratio of semi-axis \\
\hline & 4 & Tumor boundary irregularity factor \\
\hline & 5 & Fractal dimension factor \\
\hline & 6 & Area of the extracted region in pixels \\
\hline & 7 & Longest radial length \\
\hline & 8 & High intensity percentage in mass region \\
\hline & 9 & Mean intensity inside the mass region \\
\hline & 10 & Standard deviation of pixel value inside the mass region \\
\hline & 11 & Mean gradient of region boundary \\
\hline & 12 & Mean intensity difference between the extracted region and its surrounding area \\
\hline & 13 & Standard deviation of the gradient of region boundary \\
\hline & 14 & Skew of the gradient of region boundary \\
\hline & 15 & Standard deviation of pixel values in the surrounding background \\
\hline & 16 & Number of spiculations \\
\hline & 17 & Average normalized spiculation length \\
\hline & 18 & Number of spiculations with a length exceeding a given threshold \\
\hline & 19 & Spiculation angular distribution around the mass \\
\hline \multirow{9}{*}{$\begin{array}{c}\text { Global } \\
\text { Features }\end{array}$} & 20 & Average mean intensity value of right and left breast \\
\hline & 21 & Average intensity standard deviation of right and left breast \\
\hline & 22 & Average energy of right and left breast \\
\hline & 23 & Absolute difference of mean intensity value of right and left breast \\
\hline & 24 & Absolute difference of intensity standard deviation of right and left breast \\
\hline & 25 & Absolute difference of energy of right and left breast \\
\hline & 26 & Ratio of the average difference of two adjacent values in histogram \\
\hline & 27 & Ratio of the average value of histogram \\
\hline & 28 & Ratio of the standard deviation of the histogram \\
\hline
\end{tabular}

Table 2: Feature categories and descriptions

Having acquired the features from preprocessing, we can apply these features to our proposed three stage SSL algorithm. The three stages are data weighing, feature selection, and data labeling. The following paragraphs will introduce each stage respectively.

\subsection{1 stage one: data weighing}

For SSL algorithms, one key to its success lies on choosing each classifier's confident predictions based on the labeled dataset, so the influence of noisy data in the initial labeled dataset is extremely strong. The first stage in our proposed scheme is data weighing, which is rarely used in SSL or computerized cancer analysis systems. To the best of our knowledge, the data weighing function (Eq. 1) is newly proposed in our study and first time used in CAD system. The idea of data weighing is based on DROP3 instance selection method [26]; however, instead of eliminating the atypical data, we assigned different weights to different data. This stage provides an evaluation for every initial labeled data in the following steps: firstly, remove any instance $L_{i}$ misclassified by its $k$ nearest neighbors (k was set to 5 in this study), which acts as a 
noisy data filter and helps avoid overfitting; secondly, compare the prediction accuracy between removing instance $L_{i}$ and keeping instance $L_{i}$ from the original labeled dataset. The accuracy of keeping the instance is noted as $A_{k}$ and the accuracy of removing the instance is $A_{r}$, then the weight of $L_{i}$ is calculated by the following weighing function:

$$
W_{i}=e^{A_{k}-A_{r}}
$$

\subsubsection{The stage two: feature selection}

A Correlation-based Feature Selector (CFS) was implemented to select the most efficient features from the feature pool. CFS was first introduced by [26], and it ranks feature subsets according to a correlation based heuristic evaluation function:

$$
M_{s}=\frac{t \bar{r}_{c f}}{\sqrt{t+t(t+1) \bar{r}_{f f}}}
$$

$M_{s}$ is the heuristic "merit" of a feature subset with $\mathrm{t}$ features, $\bar{r}_{c f}$ is the mean feature-class correlation, and $\bar{r}_{f f}$ is the mean feature-feature intercorrelation [26]. The numerator indicates how predictive of the class a set of features are, and the denominator represents the redundancy of the features. The best first method was chosen as our search method, and the search was terminated when no improvement was observed in five consecutive feature set changes.

\subsubsection{Stage three: dividing co-training data labeling}

Our dividing co-training algorithm is designed to efficiently add the most confident unlabeled data to the labeled data pool and it is based on a graph based semi-supervised learning algorithm [27-28]. Compared to the existing algorithms [27-28], our proposed similarity function (Eq. 3) was modified and the weighted data were incorporated into the similarity function. The traditional co-training method was first introduced in the machine learning area; it trains two classifiers based on two independent attribute subsets and labels the unlabeled data whenever the two classifiers reached an agreement. However, the requirement of two sufficient and redundant attribute subsets is difficult to be satisfied in most applications. Inspired by this idea, the dividing co-training algorithm was developed in order to divide the labeled data into several equal-sized subsets instead of struggling to find two redundant feature sets. Figure 3 shows the intuitive idea of the dividing co-training algorithm.

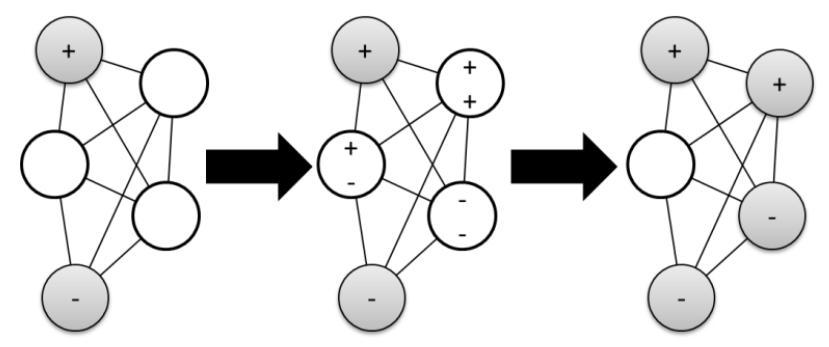

Figure 3: The process of dividing co-training data labeling method. The gray circles represent labeled data and the white circles represent unlabeled data, and the plus and minus sign denote the labels. The unlabeled data with same predictions will be added to the labeled dataset. 
It starts with a small number of labeled data $\left(L=\left\{\left(x_{i}, y_{i}\right)_{i=1}^{n_{L}}\right\}\right)$ and a larger number of unlabeled data $\left(U=\left\{\left(x_{i}\right)_{i=n_{L}+1}^{n}\right\}\right)$, where $x_{i}$ indicates the data and $y_{i}= \pm l$ shows its label. In the first step, a weighted graph is constructed with the nodes reflecting the labeled data and unlabeled data with the edges denoting the similarity between each data points. In the second step, we calculated the similarity $s_{i j}$ between two nodes $x_{i}$ and $x_{j}$ according to the computed weights in the first step:

$$
s_{i j}=\left\{\begin{array}{ll}
\exp \left(-\frac{\left(x_{i}-x_{j}\right)^{T}\left(x_{i}-x_{j}\right)}{w_{i} w_{j} a^{2}}\right) & \text { if } \mathrm{i} \neq \mathrm{j} \\
0 & \text { otherwise }
\end{array}\right\}
$$

Since no weight was available for the unlabeled data, they were initially assigned to $w_{i}=1$. And $k$-nearest-neighbors $(k N N)$ method was used to define the adjacent nodes, and the similarity of these nodes was calculated by Eq (3). As our algorithm is based on the so-called "cluster assumption" algorithm which is summarized by [29]: two points are likely to have the same class label if there is a path connecting them that passes through regions of high density only. The following quadratic objective function satisfies this assumption, and it was first introduced by [30]:

$$
\min _{f}(f-y)^{T}(f-y)+\mu f^{T} L f
$$

where $y$ is the label vector, $f$ is the prediction vector, $\mu$ is the parameter trading off loss and smoothness. $L$ is the graph Laplacian matrix which is defined as $L=D-W$, where $D=\operatorname{diag}\left(d_{i}\right), d_{i}=\sum_{i} s_{i j}$. The solution of Eq (4) is:

$$
f=(I+\mu L)^{-1} y
$$

where $I$ is identity matrix.

\subsubsection{The three stage semi-supervised learning}

Diversity among the classifiers is a major factor to determine the sensitivity of the noisy data in agreement-based algorithms [31]. The way we increase diversity is to divide initial labeled dataset into different subgroups. After each iteration the expanded labeled dataset was shuffled and re-divided to build the new classifier. However, simply splitting the original labeled group into too many subgroups would make each subgroup too small to build a reliable classifier. So in our proposed dividing co-training algorithm, we divided the original dataset into two equal-sized subgroups five times, so each subgroup was just half of the original size. The description of this method is described as follows.

Our proposed three stage SSL method is a combination of data weighing, feature selection and dividing co-training data labeling. In the first step, the labeled dataset was divided into two subgroups five times and each subgroup was used to train one classifier with the dividing co- 
training data labelling method independently. So after this step we got 10 subgroups altogether and each subgroup had its own classifier. Then, each classifier made a prediction for every unlabeled data based on Eq (5), and the data with high possibility for most classifiers (i.e. common confident data) were appended to the labeled dataset. In this study the minimum classifier amount was set to 7 . Repeat the whole process until no more common confident data appeared, the final labeled dataset was used for classifying the test data. In the iteration $t$, every time after the expanded labeled dataset was divided into two subgroups, the prediction accuracy $A c c_{t}^{c_{i}^{t}}$ of the new classifier $c_{i}^{t}$ was calculated on the combination of initial labeled data and $30 \%$ of most confident newly labeled data. And a comparison was made with the same classifier accuracy in the previous iteration $t-1$, if $A c c_{t}^{c_{i}^{t}}<A c c_{t-1}^{c_{i}^{t-1}}$; the expanded labeled dataset from previous iteration was re-divided until the prediction accuracy improved. In the proposed algorithm, not all unlabeled data have to be appended to the labeled dataset. If no agreement was reached in the end, these data were treated as noisy data or atypical data; the final labeled dataset only contains the common confident data. The flowchart is shown in Figure 4.

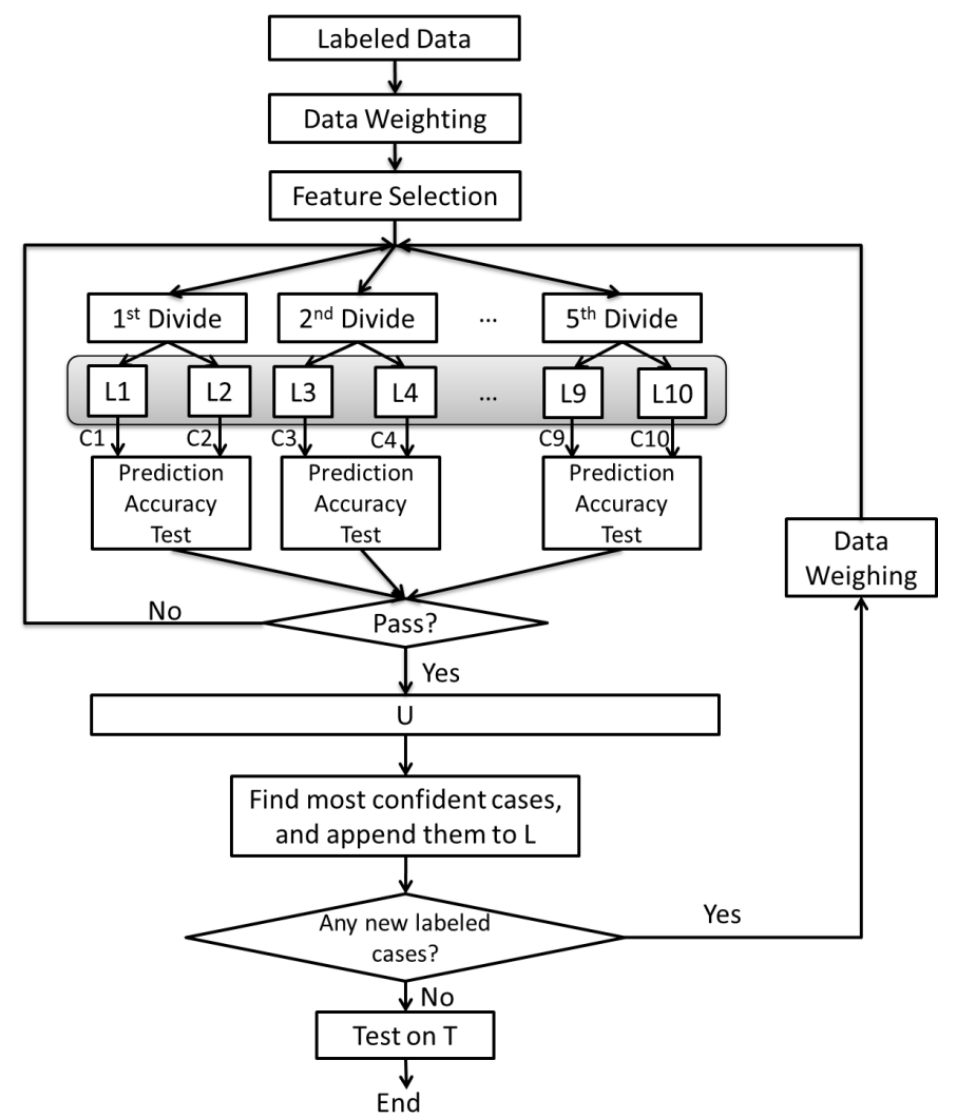

Figure 4: The flowchart of three stage SSL algorithm. ( $\mathrm{L}$ is the labeled data, $\mathrm{U}$ is the unlabeled data, $\mathrm{T}$ is the test data, $\mathrm{L}_{\mathrm{i}}$ is the subgroup of labeled data, $\mathrm{C}_{\mathrm{i}}$ is the classifier trained by dividing co-training data labeling method)

\section{Results}


In order to explore the possible impact of incorporating undiagnosed data into our CAD system and into each module of our proposed SSL scheme, different experimental configurations were considered in this study. 10-fold cross validation method was performed for evaluation. The whole process was repeated 100 times and the average difference of $\mathrm{AUC}, \triangle \mathrm{AUC}=$ $\mathrm{AUC}_{\text {label+unlabel }}-\mathrm{AUC}_{\text {label}}$, were calculated and compared. As all the data we used was labeled data, every time before we repeat the whole process we randomly removed certain number of truth files, and these data were considered as unlabeled data.

Different feature selection methods and machine learning methods were compared and evaluated, and the performance of different combinations of methods using the features extracted from the 512 ROIs were shown in Table 3. Three feature selection algorithms: information gain, classifier subset evaluator, and correlation based feature selection [26] were tested and compared. Three representative machine learning models, artificial neural network (ANN), support vector machine (SVM) and SSL, were used to perform classifications on predicting early breast cancer. The measurements were observed at the false positive rate 0.25 per image, and the unlabeled data for the SSL method is set to 360 . The selected features using different feature selection methods are listed in Table 4, and the number in bold indicate the best performance from the compared techniques.

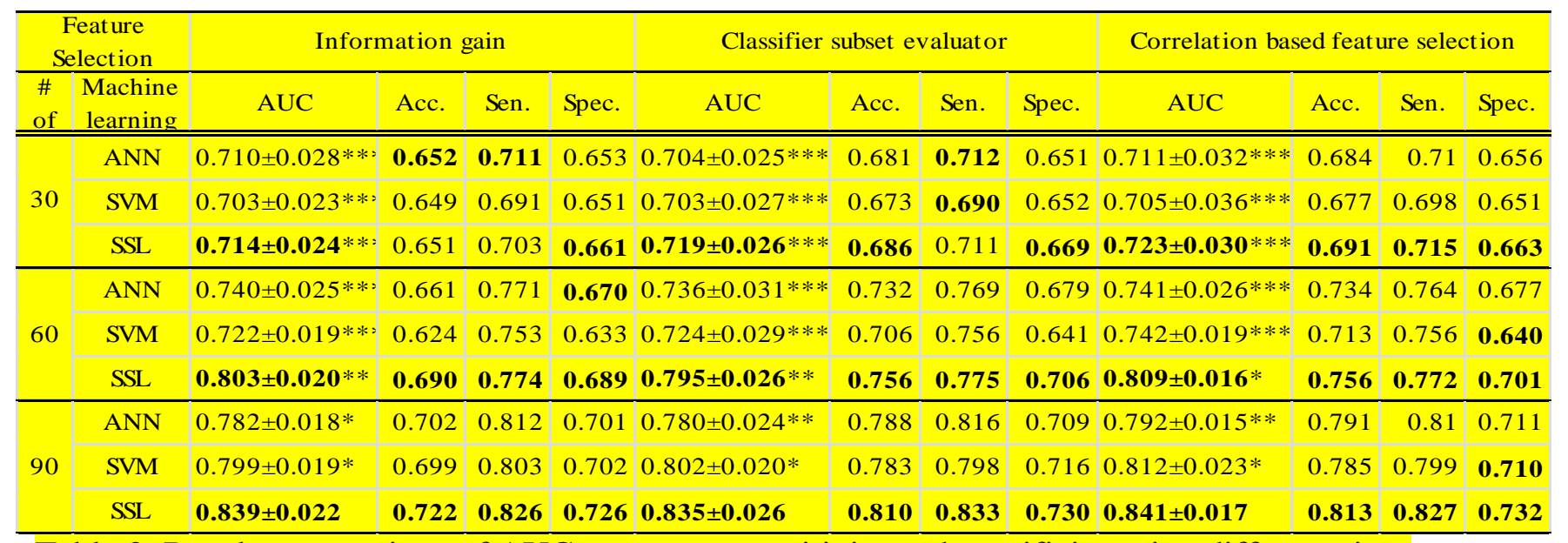

Table 3: Results comparison of AUC, accuracy, sensitivity and specificity using different sizes

of initial labeled dataset, with feature selection methods and machine learning methods. The amount of unlabeled data is set to 360 , and the false positive rate is 0.25 per image. The accuracy, sensitivity and specificity are denoted as acc., sen., and spec. All AUCs were compared with the highest AUC, and we marked the AUCs have $\mathrm{p}$ values less than 0.05 with *, p values less than 0.01 with **, p values less than 0.001 with ***.

\begin{tabular}{|c|c|}
\hline Feature Selection Method & Selected Feature in Metrics (Ref. Table 2) \\
\hline Information gain & $1,4,13,16,21$ \\
\hline Classifier subset evaluator & $1,4,11,13,21,23$ \\
\hline Correlation based feature selection & $1,4,13,16,21,23$ \\
\hline
\end{tabular}

Table 4: Selected features in different schemes

As illustrated in Table 3, the best AUC, accuracy and specificity were 0.841, 0.813, 0.732, respectively, which were obtained from the correlation based feature selection method, using our 
proposed three stage SSL scheme with the 90 labeled data. The highest sensitivity we observed was 0.833 with the classifier subset evaluator, while correlation based feature selection method produced a lower sensitivity of 0.827 . Overall, the SSL method in collaboration with the correlation based feature selection method achieved the best performance from the three machine learning methods; however, when the initial labeled dataset was of a small value, such as $\mathrm{L}=30$, the results were less stable. The correlation based feature selection method gave a slightly better performance than the other two feature selection methods most of the time. Of the three influential factors shown in Table 3, the greatest influencer was the number of labeled data, while the least influential factor was the feature selection method. We also conducted the statistic test among the three feature selection methods, the lowest $\mathrm{p}$ value is 0.42 using paired $\mathrm{t}$-test, which is calculated between classifier subset evaluator and correlation based feature selection. So there is no significant differences among the three feature selection methods, but removing the feature selection modules decreased accuracies and AUCs.

To conduct a qualitatively demonstrated impact of the different sizes of labeled data and unlabeled data, we employed a metric of $\triangle \mathrm{AUC}$ and accuracy using different sizes of labeled and unlabeled datasets (Table 5). Using our proposed scheme, the highest AUC observed in our research was $0.841 \pm 0.017$ when the 90 labeled data and all unlabeled data were included. This was $7.4 \%$ higher than using the 90 labeled data only $(0.783 \pm 0.031)$. An intuitive representation of the tendency that occurs from using different sizes of the dataset is presented in Figure 5. The figure shows that the $\triangle \mathrm{AUC}$ increases with the number of unlabeled data; this increase is prominent when nearest at the vertical axis and is stablized when the the number of unlabeled data is larger. Figure 6, on the other hand, shows the increase of the $\triangle \mathrm{AUC}$ when different sizes of labeled data were used. The $\triangle \mathrm{AUC}$ for labeled data reached its peak when the amount of labeled data was around 60 .

\begin{tabular}{|c|c|c|c|c|c|c|c|}
\hline $\begin{array}{c}\text { Number } \\
\text { of } \\
\text { labeled } \\
\text { cases (L) }\end{array}$ & $\begin{array}{c}\text { Number of } \\
\text { unlabeled } \\
\text { cases (U) }\end{array}$ & $\begin{array}{c}\text { AUC } \\
\text { with L } \\
\text { only }\end{array}$ & $\begin{array}{c}\text { AUC } \\
\text { with L } \\
\text { and U }\end{array}$ & $\begin{array}{c}\text { Mean } \\
\Delta \text { AUC }\end{array}$ & $\begin{array}{c}\text { Accuracy } \\
\text { with L } \\
\text { only }\end{array}$ & $\begin{array}{c}\text { Accuracy } \\
\text { with L } \\
\text { and U }\end{array}$ & $\begin{array}{c}\text { Mean } \\
\text { Accuracy } \\
\text { Change } \\
(\%)\end{array}$ \\
\hline 30 & 50 & $0.689 * * *$ & $0.706 * * *$ & 0.017 & 0.665 & 0.672 & 1.052 \\
\hline 30 & 100 & $0.689 * * *$ & $0.712 * * *$ & 0.023 & 0.665 & 0.682 & 2.556 \\
\hline 30 & 200 & $0.689 * * *$ & $0.720 * * *$ & 0.031 & 0.665 & 0.688 & 3.458 \\
\hline 30 & 360 & $0.689 * * *$ & $0.723 * * *$ & 0.034 & 0.665 & 0.691 & 3.909 \\
\hline 60 & 50 & $0.722 * * *$ & $0.774 * * *$ & 0.052 & 0.702 & 0.731 & 4.131 \\
\hline 60 & 100 & $0.722 * * *$ & $0.789 * * *$ & 0.067 & 0.702 & 0.743 & 5.840 \\
\hline 60 & 200 & $0.722 * * *$ & $0.803 * * *$ & 0.081 & 0.702 & 0.752 & 7.122 \\
\hline 60 & 360 & $0.722 * * *$ & $0.809 * *$ & 0.087 & 0.702 & 0.756 & 7.692 \\
\hline 90 & 50 & $0.783 * * *$ & $0.816 *$ & 0.033 & 0.768 & 0.783 & 1.953 \\
\hline 90 & 100 & $0.783 * * *$ & $0.827 *$ & 0.044 & 0.768 & 0.794 & 3.385 \\
\hline 90 & 200 & $0.783 * * *$ & 0.836 & 0.053 & 0.768 & 0.808 & 5.208 \\
\hline 90 & 360 & $0.783 * * *$ & 0.841 & 0.058 & 0.768 & 0.813 & 5.859 \\
\hline
\end{tabular}

Table 5: The comparison of different amounts of labeled data and unlabeled data. The groups with highest AUC was set as the reference, and all the tests are paired t-test. The AUCs with $p$ values less than $0.05,0.01$ and 0.001 are marked with $*, * *, * * *$. 

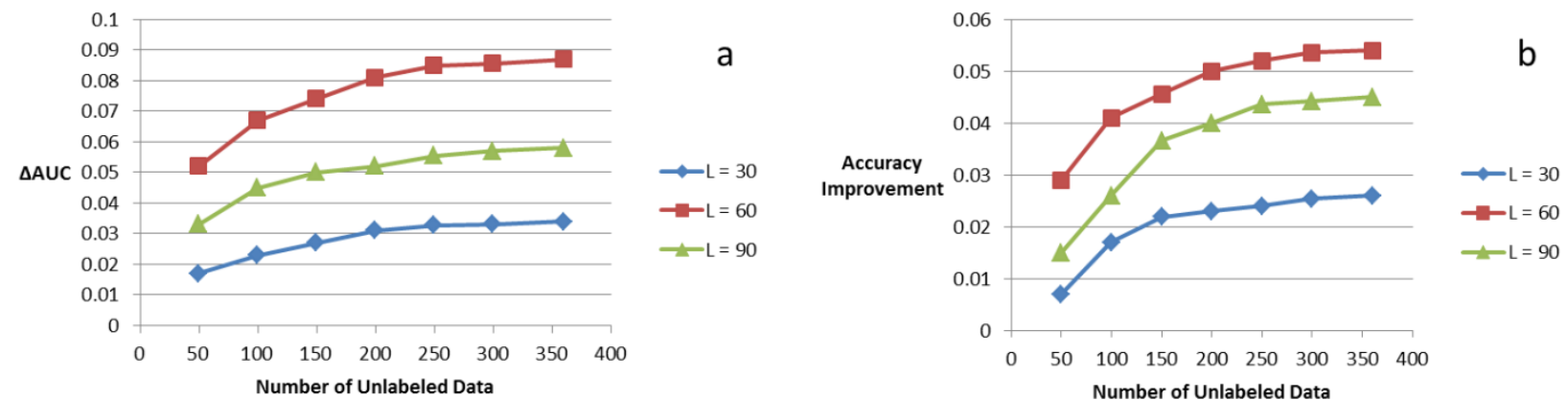

Figure 5: (a) Average $\triangle \mathrm{AUC}$ at different sizes of unlabeled data (b) average accuracy improvement with the change of different sizes of unlabeled data
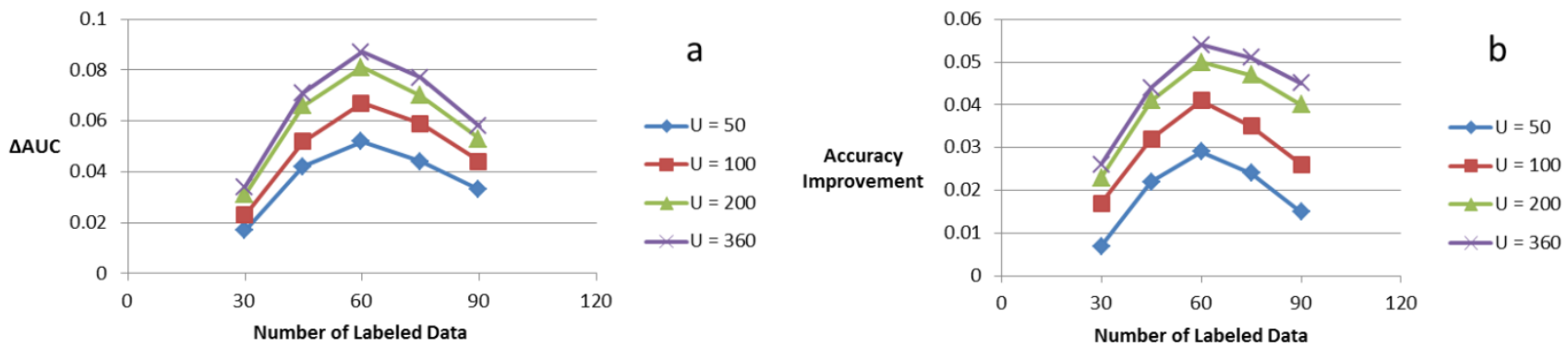

Figure 6: (a) $\triangle \mathrm{AUC}$ improvement with the change of different sizes of labeled data (b) accuracy improvement with the change of different sizes of labeled data

To illustrate the necessity of each component in the proposed method, we developed several other schemes and compared their performances. Scheme 1 is our originally proposed scheme; Scheme 2 included the local features only; Scheme 3 included the local features only and also labeled data only; Scheme 4 had the data weighing module removed; Scheme 5 included labeled data only; Scheme 6 used ANN; Scheme 7 used SVM. Figure 7 compares the differences in terms of $\triangle \mathrm{AUC}$, accuracy, sensitivity and specificity (measured at the false positive rate 0.25 per image). The AUC was calculated at varying numbers of labeled data and 360 unlabeled data. 

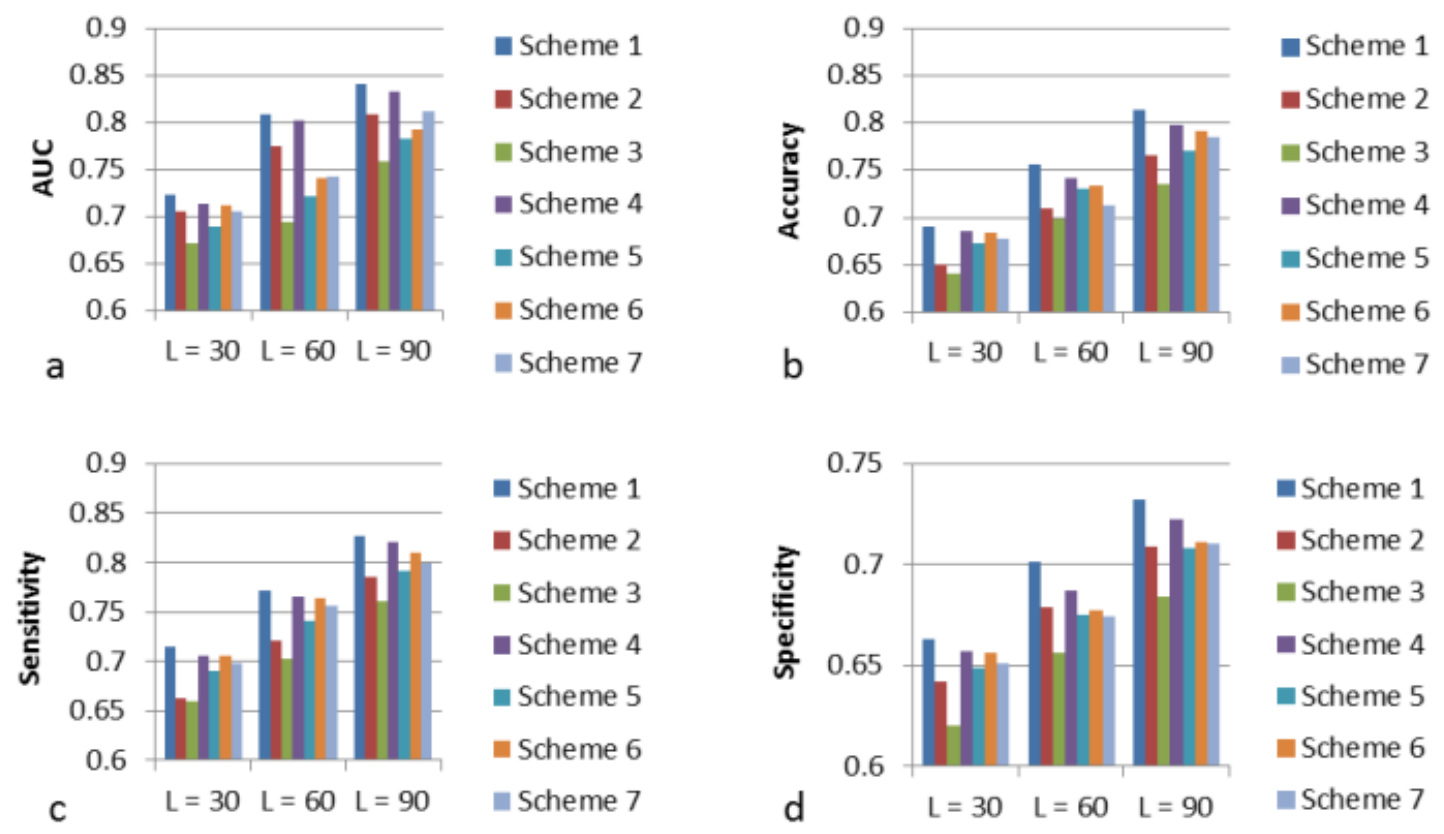

Figure 7: Results comparison of different schemes and different sizes of labeled data ( $\mathrm{L}$ is the size of labeled data) in terms of (a) AUC (b) accuracy (c) sensitivity (d) specificity

From the comparison, Scheme 1 always generates the best performance in terms of AUC, accuracy, sensitivity and specificity. And Scheme 4 has the second best performance, and Scheme 3 is the worst. From the comparison of Scheme 1 and Scheme 2, we found that using density asymmetry features brought a $6.1 \%$ improvement in accuracy and a $5.4 \%$ improvement in sensitivity when using 90 labeled data. From the comparison of Scheme 1 and Scheme 4, it was noted that data weighing has less influence in sensitivity (1.0\% average improvement) than specificity (1.4\% average improvement). From the comparison of Scheme 3 and Scheme 4, density asymmetry features have more influence on data weighing. We also conducted the ANOVA test for all the seven schemes using 90 labeled data and all unlabeled data, and the results reject the null hypothesis at the critical value of 0.05 .

For each scheme, we also compared the classification results of training data and test data. The average error rates were calculated for both training set and testing set in 10 fold cross validation. All the experiments were conducted with 90 initial labeled data and all unlabeled data, and the results are shown in Table 6 . From the results, scheme 1 (originally proposed scheme) has the lowest average error rate in testing set, but scheme 4 (with no data weighing module) has the lowest average error rate in training set. This shows data weighing effectively reduced the overfitting risk in our proposed scheme.

\begin{tabular}{|c|c|c|}
\hline Average error rate & Training set & Testing set \\
\hline Scheme 1 & 0.160 & 0.187 \\
\hline Scheme 2 & 0.209 & 0.233 \\
\hline
\end{tabular}




\begin{tabular}{|l|l|l|}
\hline Scheme 3 & 0.220 & 0.264 \\
\hline Scheme 4 & 0.144 & 0.202 \\
\hline Scheme 5 & 0.178 & 0.229 \\
\hline Scheme 6 & 0.169 & 0.209 \\
\hline Scheme 7 & 0.173 & 0.216 \\
\hline
\end{tabular}

Table 6: Average error rate of training set and test set.

To gain insight into the learning process of the developed dividing co-training method, the average error rates at each learning iteration were further averaged over different unlabeled data rates. Figure 8 shows the averaged changes in the number of newly labeled data and the accuracy as the iterations proceeded.
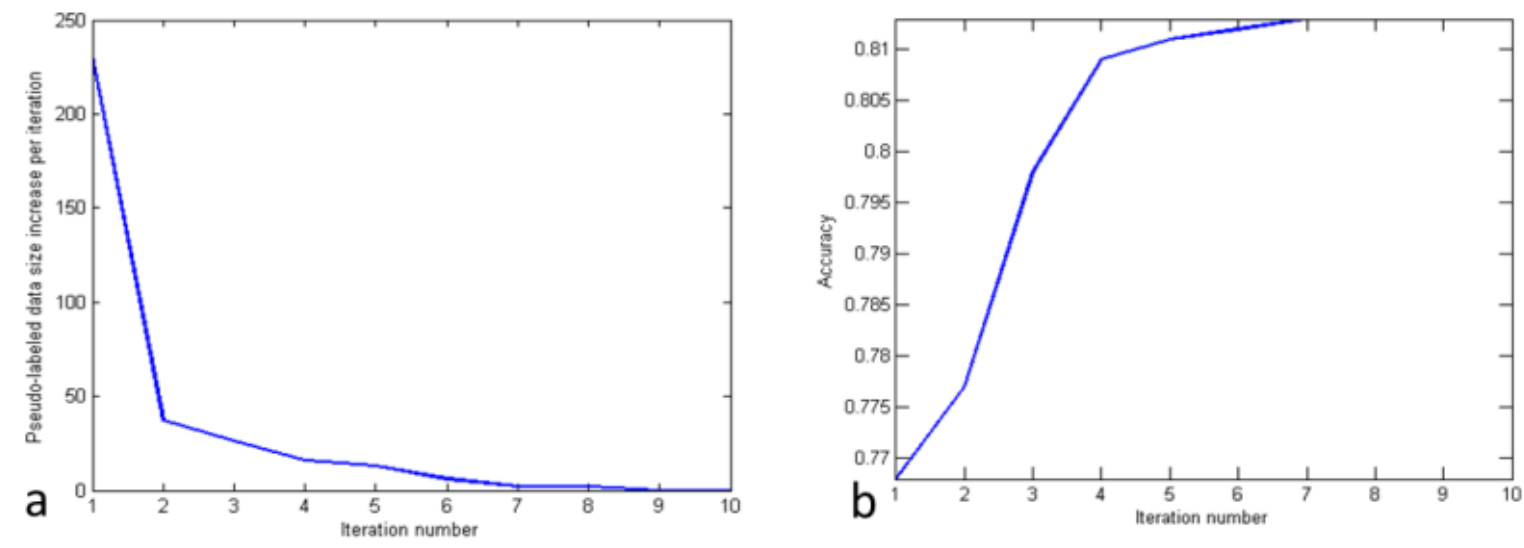

Figure 8: Changes during the dividing co-training iterations (a) number of newly labeled data and (b) accuracy

To further test our scheme, we applied the pre-trained system to all the 3142 ROIs excluding the samples already being used for training. This resulted in the case-based and region-based FROC curves below (Figure 9). Both case- and region- based FROC curves detection sensitivity levels were analyzed at a rate of 0.25 false positive per image. In the case-based analysis, a positive case was defined when at least one mass region was detected on one mammogram, and the region-based analysis was defined when all ROIs were counted independently. 

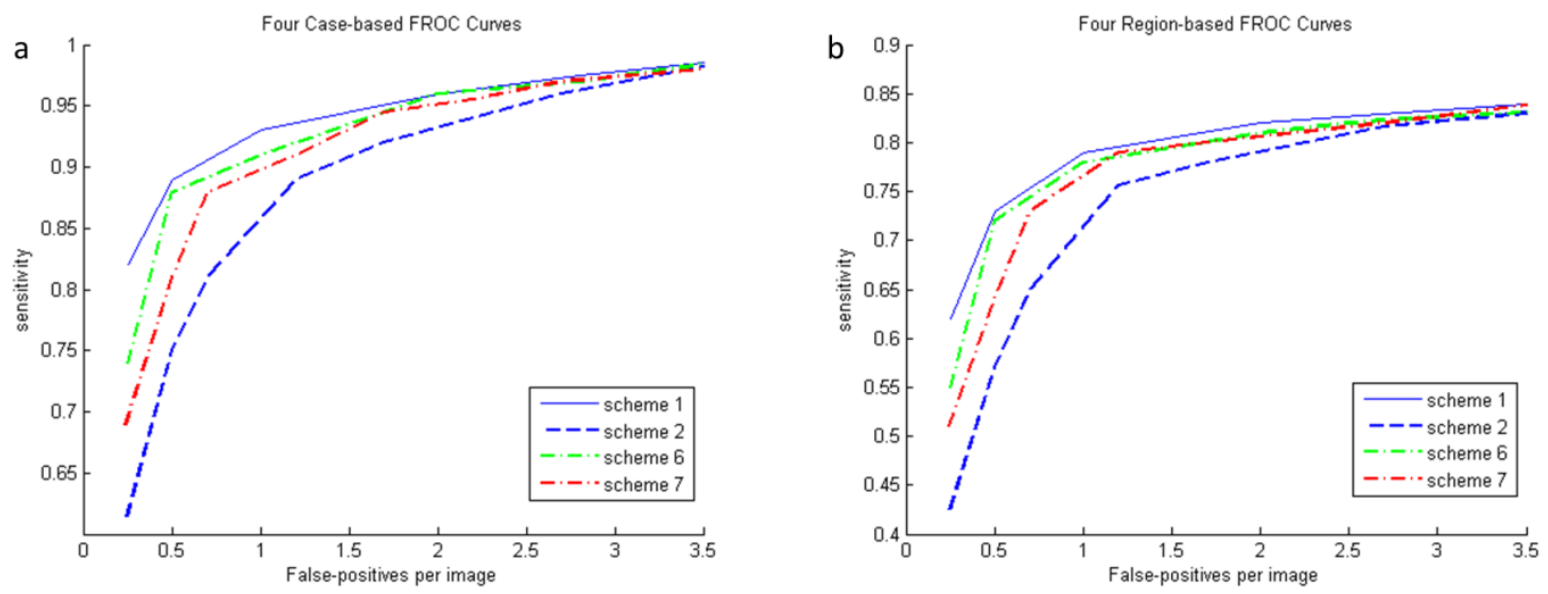

Figure 9: (a) Comparison of case-based FROC curves representing the performance of our proposed schemes, the performance of our proposed scheme with local features only, the performance of ANN and SVM. (b) comparison of region-based FROC curves representing the performance of our proposed schemes, the performance of our proposed scheme with local features only, the performance of ANN and SVM.

When incorporating all the ROIs into our system, the proposed features can help CAD have a better performance; this is shown on the region-based FROC curve and the case-based FROC curve (Figure 9). The region-based and cased-based sensitivity of our scheme were 0.5813 and 0.7678 at the rate of 0.25 false-positive per image, while the removal of global features lead to the corresponding sensitivity dropped down to 0.4659 and 0.6574 . The SVM and ANN based schemes also had the same tendency. In the end we removed the covered labels for all unlabeled data, so we have 3142 labeled ROIs from 400 cases with corresponding truth files. For the comparison reason, we used the same 512 ROIs, extracted the same features and applied feature selection method with SVM classifier to them. The observed AUC was $0.866 \pm 0.011$.

\section{Discussion}

We have described a three stage semi-supervised learning approach to improve the overall performance of a computerized breast cancer analysis system with unlabeled data. To the best of our knowledge the three stage SSL CAD system, which includes dividing co-training method designed to split the initial labeled dataset and data weighing for labeled data optimization, combined with the computerized breast cancer analysis system was first presented in this paper, and no similar method has been reported in the area of computerized breast cancer detection. In this paper we also tested the feasibility of combining the local and global features together for mass detection, designed the data weighing function and the modified the graph-based SSL similarity function. The advantages of the proposed method for mass detection over the conventional approach of only utilizing labeled data are: (1) the abundance of available unlabeled data can be used in this scheme; (2) this algorithm has acceptable sensitivity even at a low false positive rate.

Using our proposed scheme, the highest AUC observed in our research was 0.841 when including 90 labeled data and all unlabeled data. This was $7.4 \%$ higher than using labeled data 
only. Based on the comparison of different amounts of unlabeled data (Table 5 and Figure 5), it can be concluded that the size of unlabeled data has a significant impact on the performance of the system. The influence was especially strong when the size was small and the influence becomes limited when the size was incremented. From the comparison of different sizes of initial labeled data (Table 5 and Figure 6), it can be noted that the AUC grows with the increase of labeled data. This means a larger labeled dataset provides better performance for both the traditional method and the proposed method. However the AUC difference grew when the labeled data was small and peaked when the labeled data was around 60; after this peak the AUC difference began to drop (Figure 6). This demonstrates our proposed approach has its advantages when labeled data is insufficient (less than 100 in our study), and the difference being most significant when the amount of labeled data was close to 60 . This is probably because of two factors: 1) our proposed scheme is less sensitive to initial labeled data than supervised learning based schemes, as it has the ability to use the information from the unlabeled data to improve its performance 2) the limitation of insufficient labeled data will become more obvious if the size of labeled data is extremely small (like 30 in our study), the performance improvement of using unlabeled data would become limited. Since our algorithm requires splitting the initial labeled dataset, if the size is extremely small the split dataset will be even smaller which makes the scheme more sensitive to noise [31-32].

The following three major parts compose our proposed scheme: data weighing, feature selection and dividing co-training data labeling. Among the three modules in the system, dividing cotraining data labeling stage is the most important stage. It made our algorithm can utilize the unlabeled data, which is a significant difference from other existing CAD system. As illustrated in Table 3, SSL method generally achieved the best performance (in terms of accuracy, AUC, sensitivity, specificity) from the three machine learning methods. The correlation based feature selection method gave a slightly better performance in comparison to the other two feature selection methods most of the time. From among the three influence factors in Table 3, the number of the initial labeled data has the strongest influence, and the feature selection method has the least influence. Data weighing is the first time introduced in CAD system, and Figure 7 demonstrates the contribution of this module; removing the data labeling module dramatically decrease the specificity (Figure 7d); removing the global density features leads to the drop of sensitivity (Figure 7c); the correlation based feature selection module is the most suitable feature selection method among all the tested feature selection methods (Table 3); our proposed SSL scheme shows a better AUC and accuracy than the supervised methods (ANN and SVM) (Figure 7a,b, Table 5). This study also demonstrated that bilateral mammographic density asymmetry is not totally correlated with the ROI features, and can be incorporated as a supplement for our CAD scheme. And this also agrees with our assumption that the considering the information from surrounding areas of the suspicious region can improve the prediction performance.

For all co-training style systems, the base classifier should be both accurate and diverse, but it is noted that diversity usually decreases as the iteration proceeds [33]. Because the exploitable diversity depends on the given dataset and classifiers, there frequently seems to be a conflict between diversity and accuracy. Intuitively, the more accurate the classifiers are, the more similar their predictions are likely to be [16]. However, with the newly labeled data, manipulating the training set could be a feasible way to recreate diversity. Therefore, as a strategy similar to inter-training [16], we resampled 10 different subsets from the original labeled dataset and newly added pseudo-labeled dataset, and the more confident data will be given 
higher weights in each iteration. Furthermore, the prediction accuracy test has been made to roughly estimate the performance of the newly trained $c_{i}$ which refined the labeled dataset. In this way our scheme can keep improving the accuracy every round while retaining the diversity.

Moreover, we re-designed the CAD system for mass detection, and the mammographic tissue density information was incorporated into our proposed schemes. Bilateral mammographic density asymmetry features have the ability to reduce the false positive rate [4], and based on our previous study [21] we included more features to the proposed scheme. From the comparison of whether using or not using the global density asymmetry features we noticed that these features have a significant influence on performance, especially in accuracy and sensitivity (Figure 7). Accuracy improves $6.1 \%$ and sensitivity improves 5.4\% when using the 90 labeled data and all unlabeled data. From Figure 9, the region-based and cased-based sensitivity of our scheme were increased $24.8 \%$ and $16.8 \%$, respectively, at the rate of 0.25 false-positive per image when added global features.

\section{Conclusion}

In summary, this study demonstrated that our proposed three stage semi-supervised learning can improve the CAD performance by using unlabeled data. As a result, incorporating unlabeled data to computerized cancer scheme may have a significant impact for computerized breast cancer research. However, we would like to stress that unlabeled data could never replace labeled data, it is only a supplement. This is because labeled data contains more accurate information from the radiologist, and the same amount of labeled data usually gives better performance than the same amount of mixed dataset (labeled data and unlabeled data). Despite this fact, this methodology can be used in preliminary studies especially when the labeled data is limited. Instead of being hindered by the dataset, considering the unlabeled data, which is usually ignored, can be an alternative option.

\section{Acknowledgements}

This work was partially supported by National Institutes of Health (SC1CA166016), National Science Foundation (DUE-TUES-1246050) and Department of Education (Award \#P031S120131). We also thank the Border Biological Research Center (BBRC) Core Facilities at The University of Texas at El Paso (UTEP) for their support, which were funded by RCMINIMHD-NIH grant (8G12MD007592). 


\section{References}

[1] Siegel, R., Naishadham, D., \& Jemal, A. (2013). Cancer statistics, 2013. CA: a cancer journal for clinicians, 63(1), 11-30.

[2] Smith, R. A., Cokkinides, V., Brooks, D., Saslow, D., Shah, M., \& Brawley, O. W. (2011). Cancer screening in the United States, 2011. CA: A Cancer Journal for Clinicians, 61(1), 8-30.

[3] Madigan, M. P., Ziegler, R. G., Benichou, J., Byrne, C., \& Hoover, R. N. (1995). Proportion of breast cancer cases in the United States explained by well-established risk factors. Journal of the National Cancer Institute, 87(22), 1681-1685.

[4] Wang, X., Li, L., Xu, W., Liu, W., Lederman, D., \& Zheng, B. (2012). Improving performance of computer-aided detection of masses by incorporating bilateral mammographic density asymmetry: an assessment. Academic Radiology, 19(3), 303-310.

[5] Shiraishi, J., Pesce, L. L., Metz, C. E., \& Doi, K. (2009). Experimental design and data analysis in receiver operating characteristic studies: lessons learned from reports in radiology from 1997 to 2006. Radiology, 253(3), 822.

[6] Jamieson, A. R., Giger, M. L., Drukker, K., \& Pesce, L. L. (2010). Enhancement of breast CADx with unlabeled data. Medical physics, 37, 4155.

[7] Kim, J., \& Shin, H. (2013). Breast cancer survivability prediction using labeled, unlabeled, and pseudo-labeled patient data. Journal of the American Medical Informatics Association, 20(4), 613-618.

[8] Blum, A., \& Mitchell, T. (1998, July). Combining labeled and unlabeled data with cotraining. In Proceedings of the eleventh annual conference on Computational learning theory (pp. 92-100). ACM.

[9] Kiritchenko, S., \& Matwin, S. (2011, November). Email classification with co-training. In Proceedings of the 2011 Conference of the Center for Advanced Studies on Collaborative Research (pp. 301-312). IBM Corp.

[10] Zhao, Y. H., Wang, H. L., \& Cui, R. Y. (2012). Abnormal Voice Detection Algorithm Based on Semi-supervised Co-training Algorithm. Advanced Materials Research, 461, 117-122.

[11] Wan, X. (2009, August). Co-training for cross-lingual sentiment classification. In Proceedings of the Joint Conference of the 47th Annual Meeting of the ACL and the 4th International Joint Conference on Natural Language Processing of the AFNLP: Volume 1Volume 1 (pp. 235-243). Association for Computational Linguistics.

[12] Li, M., \& Zhou, Z. H. (2007). Improve computer-aided diagnosis with machine learning techniques using undiagnosed samples. Systems, Man and Cybernetics, Part A: Systems and Humans, IEEE Transactions on, 37(6), 1088-1098.

[13] Sun, W., Tseng, T. L., Zheng, B., Lure, F., Wu, T., Francia, G., ... \& Qian, W. (2014, March). Using undiagnosed data to enhance computerized breast cancer analysis with a three 
stage data labeling method. In SPIE Medical Imaging (pp. 90350T-90350T). International Society for Optics and Photonics.

[14] Zheng, B., Ganott, M. A., Britton, C. A., Hakim, C. M., Hardesty, L. A., Chang, T. S., ... \& Gur, D. (2001). Soft-Copy Mammographic Readings with Different Computer-assisted Detection Cuing Environments: Preliminary Findings 1. Radiology, 221(3), 633-640.

[15] US Preventive Services Task Force. (2009). Screening for breast cancer: US Preventive Services Task Force recommendation statement. Annals of Internal Medicine, 151(10), 716.

[16] Jiang, Z., Zeng, J., \& Zhang, S. (2013). Inter-training: Exploiting unlabeled data in multiclassifier systems. Knowledge-Based Systems, 45, 8-19.

[17] Olvera-López, J. A., Carrasco-Ochoa, J. A., Martínez-Trinidad, J. F., \& Kittler, J. (2010). A review of instance selection methods. Artificial Intelligence Review, 34(2), 133-143.

[18] Brighton, H., \& Mellish, C. (2002). Advances in instance selection for instance-based learning algorithms. Data mining and knowledge discovery, 6(2), 153-172.

[19] Sun, X., Qian, W., \& Song, D. (2004). Ipsilateral-mammogram computer-aided detection of breast cancer. Computerized Medical Imaging and Graphics, 28(3), 151-158.

[20] Lihua, L., Qian, W., \& Clarke, L. P. (1997). Digital mammography: computer-assisted diagnosis method for mass detection with multiorientation and multiresolution wavelet transforms. Academic radiology, 4(11), 724-731.

[21] Sun, W., Zheng, B., Lure, F., Wu, T., Zhang, J., Wang, B. Y., ... \& Qian, W. (2014). Prediction of near-term risk of developing breast cancer using computerized features from bilateral mammograms. Computerized Medical Imaging and Graphics, 38(5), 348-357.

[22] Qian, W., Sun, X., Song, D., \& Clark, R. A. (2001). Digital mammography: Wavelet transform and Kalman-filtering neural network in mass segmentation and detection. Academic Radiology, 8(11), 1074-1082.

[23] Qian, W., Li, L., \& Clarke, L. P. (1999). Image feature extraction for mass detection in digital mammography: Influence of wavelet analysis. Medical Physics, 26, 402.

[24] Wolfe JN. Breast patterns as an index of risk for developing breast cancer. Am J Roentgenol AJR 1976; 126:1130-1139.

[25] Boyd NF, Martin IJ, Stone J, et al. Mammographic densities as a marker of human breast cancer risk and their use in chemoprevention. Curr Oncol Rep 2001; 3:314-321.

[26] Hall, M. A. (1999). Correlation-based feature selection for machine learning (Doctoral dissertation, The University of Waikato).

[27] He, J., Carbonell, J. G., \& Liu, Y. (2007, January). Graph-Based Semi-Supervised Learning as a Generative Model. In IJCAI (pp. 2492-2497). 
[28] Park, K., Ali, A., Kim, D., An, Y., Kim, M., \& Shin, H. (2013). Robust predictive model for evaluating breast cancer survivability. Engineering Applications of Artificial Intelligence, 26(9), 2194-2205.

[29] Chapelle O.,WestonJ.,SchölkopfB.,2003.Clusterkernelsforsemi-supervised learning. In: Advances in Neural Information Processing Systems, The MIT Press, Cambridge, England, pp.585-592.

[30] Choi, I., Park,K., Shin,H., 2008. Sharpened graph ensemble for semi-supervised learning. Intell. Data Anal. 17,387-398.

[31] Wu H, Miao Z, Wang Y, et al. Optimized recognition with few instances based on semantic distance[J]. The Visual Computer, 2015, 31(4): 367-375.

[32] Wu H, Miao Z, Wang Y, et al. Image completion with multi-image based on entropy reduction[J]. Neurocomputing, 2015, 159: 157-171.

[33] Krogh, A., \& Vedelsby, J. (1995). Neural network ensembles, cross validation, and active learning. Advances in neural information processing systems, 231-238. 